\title{
A Study of Rehabilitation of Speech in Post-laryngectomy Cases, as Seen in a Tertiary Care Institution of Esophageal Speech vs Speech with Tracheoesophageal Puncture Prosthesis
}

\author{
X Anton $\operatorname{Dev}^{1}$, Somnath Saha ${ }^{2}$
}

\begin{abstract}
Aim: To assess the preference of the different modalities of post-laryngectomy speech rehabilitation in patients and review the outcome parameters of esophageal speech and tracheoesophageal puncture (TEP) prosthesis modalities in comparison with the corresponding existing data.

Materials and methods: A longitudinal prospective study conducted over a period of 1 year in the Department of ENT and Head-Neck Surgery in a tertiary care hospital. Patients of advanced laryngeal malignancies that were planned for total laryngectomy were included in this surgery. Based on the patients' choice different modalities were adopted and the patients were followed up with regular training. Maximum takers were for esophageal speech followed by TE puncture and prosthesis insertion, and the outcomes in these two modalities were evaluated in detail. Six outcome parameters were assessed for the patients and consolidated. Then they were compared with the corresponding values obtained from previous studies to arrive at the results.

Results: A total of ten cases of total laryngectomy was studied during the period of study. It was found that greater patient preference was for the TEP prosthesis modality. The mean frequency of phonation, mean intensity for vowels, and word production per minute in the TEP prosthesis were almost $80 \%$ of that seen in the esophageal prosthesis group. The most significant difference was in mean maximum phonation time which was only $37 \%$ of that seen in the TEP prosthesis group.

Conclusion: In the present scenario until more advanced modalities of post-laryngectomy speech rehabilitation become more easily accessible to and affordable for the economically weaker sections of the society, esophageal speech modality is a dependable alternative. Further with proper training and follow-up, results with esophageal prosthesis are promising.

Keywords: Esophageal speech, Post-laryngectomy, Tracheoesophageal puncture prosthesis.

International Journal of Phonosurgery \& Laryngology (2019): 10.5005/jp-journals-10023-1170
\end{abstract}

\section{INTRODUCTION}

In today's scenario despite the ongoing advances in conservative management of laryngeal malignancies, total laryngectomy either as primary total laryngectomy or as salvage total laryngectomy, remains an essential management modality in advanced laryngeal malignancies.

It was first performed by Billroth in $1873 .^{1}$ The five-year disease-free survival rate was found to be $60.9 \%$ for primary total laryngectomy, 54.3\% for STL after partial laryngectomy, 50\% for salvage surgery after radiotherapy, and $43.8 \%$ for salvage surgery after chemoradiotherapy. ${ }^{2}$

It is curative as well as palliative. Larynx is the second most common site for cancer in the whole of aero-digestive tract. The most common malignancy affecting larynx is squamous cell carcinoma. After total laryngectomy there is a profound alteration in the life style of a patient. The patient is unable to swallow normally, associated with profound changes in the pattern of respiration. Olfaction is also affected.

The requirements of normal phonation are:

- Active respiratory support

- Adequate glottic closure

- Normal mucosal covering of the vocal cord

- Adequate control of vocal fold length and tension.

The vibrations of the vocal folds are complex in nature and are known as the glottic cycle. This cycle involves glottic opening and closing at set frequencies determined by the subglottic air pressure.
1,2Department of ENT and Head-Neck Surgery, Calcutta National Medical College and Hospital, Kolkata, West Bengal, India

Corresponding Author: Somnath Saha, Department of ENT and HeadNeck Surgery, Calcutta National Medical College and Hospital, Kolkata, West Bengal, India, Phone: +91 9830642186, e-mail: sahasomnath11@ gmail.com

How to cite this article: Anton Dev X, Saha S. A Study of Rehabilitation of Speech in Post-laryngectomy Cases, as Seen in a Tertiary Care Institution of Esophageal Speech vs Speech with Tracheoesophageal Puncture Prosthesis. Int J Phonosurg Laryngol 2019;9(2):36-39.

Source of support: Nil

Conflict of interest: None

Normal vocal folds produce three typical vibratory patterns: (1) falsetto, (2) modal voice (3) glottal fry in falsetto or light voice.

The mucosal wave is an important physiologic parameter during phonation. The mucosal wave is an undulation which occurs over the vocal fold mucosa and travels in an inferosuperior direction. The speed of mucosal wave ranges from 0.5 to $1 \mathrm{~m} / \mathrm{second}$. The symmetry of these mucosal waves must also be taken into consideration while studying the physiology of voice production. Any mild asymmetry between the two vocal folds must be considered as pathological.

\section{Methods of Alaryngeal Speech}

There are three methods of alaryngeal speech. They include (1) esophageal speech, (2) electrolarynx, and (3) neoglottic reconstruction/shunt procedures. 


\section{Esophageal Speech}

Most patients after total laryngectomy acquire a certain degree of esophageal speech (Table 1). In fact all the other alaryngeal speech modalities are compared with those of esophageal speech. It was the gold standard for post-laryngectomy speech rehabilitation methods during early times.

Power source-cervical esophageal segment $(80 \mathrm{~mL})$ Vibratory source-P.E. segment of pharynx (pseudoglottis) Techniques:

- Inhalational method

- Injection method

Nearly $40 \%$ of patients fail to acquire esophageal speech even after prolonged training.

Common causes of failure to develop esophageal voice are:

- Presence of cricopharyngeal spasm

- Disorders involving pharyngoesophageal segment

- Poor motivation on the part of the patient.

Cricopharyngeal myotomy must be performed in patients with cricopharyngeal spasm. If this fails botulinum toxin can be injected into the cricopharyngeal muscle. ${ }^{3,4}$ Thirty units of botulinum toxin is injected via the anterior portion of the neck (via the tracheostoma over the posterior pharyngeal wall bulge).

\section{Electrolarynx}

Electrolarynx (Fig. 1): These are electrical vibrating devices which are to be held in the submandibular region. Muscular contraction

Table 1: A comparison of the features of esophageal speech and tracheoesophageal puncture prosthesis

\begin{tabular}{ll}
\hline Esophageal speech & TEP prosthesis \\
\hline $\begin{array}{l}\text { Natural substitute voice that is } \\
\text { cost-effective }\end{array}$ & $\begin{array}{l}\text { Acquired post voice } \\
\text { rehabilitation surgery and } \\
\text { expensive for patients } \\
\text { Hands are free during use (Fig. 2) } \\
\text { Hands are not free during use } \\
\text { (Fig. 3) } \\
\text { Good intensity and good F0 } \\
\text { (120-170 Hz) }\end{array}$ \\
$\begin{array}{l}\text { Low intensity, limited } \\
\text { modulation and discontinuous }\end{array}$ \\
$\begin{array}{l}\text { Speech acquisition: 26-70\% } \\
\text { Intelligibility poor at the start, } \\
\text { but improves with training }\end{array}$ & \begin{tabular}{l} 
Intelligibility better \\
\hline
\end{tabular}
\end{tabular}

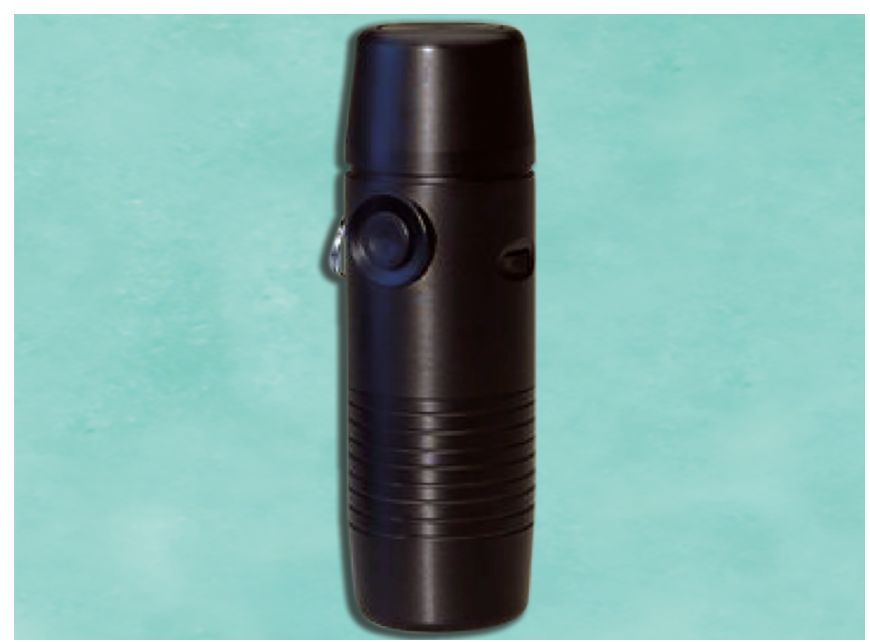

Fig. 1: Electrolarynx and facial tension can be modified to generate rudiments of speech The initial training phase to use this machine must begin even before the surgical removal of larynx. This helps the patient in easy acclimatization after surgery.

Three types of electro larynges are available. ${ }^{5}$ They are:

- Pneumatic-Dutch speech aid, Tokyo artificial speech aid etc.

- Neck (commonly used)

- Intraoral type

The major disadvantage of the electrolarynx is their mechanical quality of speech. There is also a certain degree of stomal noise. With practice a patient can reduce stomal noise by placing fingers over the stoma during phonation. These instruments are expensive and need to be maintained.

Voice restoration surgeries in patients who have undergone laryngectomy are: (1) neoglottic reconstruction; (2) shunt techniques.

\section{Neoglottic Reconstruction}

In the early days there were several attempts to develop a reliable tracheohyoidpexy procedure which could restore voice function in patients who has undergone total laryngectomy. Most of these techniques were abandoned due to complications. ${ }^{6}$

\section{Shunt Technique}

This technique involves the creation of a shunt between trachea and esophagus. This technique was first developed by Guttman in 1930.

Types of shunts:

- High tracheoesophageal shunt (Barton)

- Low tracheoesophageal shunt (Staferri)

- TEP shunts (Guttman)

Creation of a shunt between trachea and esophagus usually failed because of (1) aspiration through the fistula or (2) closure of fistula.

\section{Tracheoesophageal Puncture}

This procedure for restoration of speech in patients who have undergone total laryngectomy was first introduced by Blom and Singer in $1979 .^{8}$

Tracheo-esophageal puncture (TEP) can be performed either immediately after laryngectomy (primary TEP) or 6 weeks following successful laryngectomy (secondary TEP). It should be stressed that radiotherapy poses no threat to TEP. This procedure initially was reserved for patients who have failed to acquire esophageal speech even after prolonged efforts and are displeased with the voice produced by the artificial larynx. Currently primary TEP is becoming a preference as a first-line modality. The major problem with silicon prosthesis is candida growth. ${ }^{9}$

\section{Materials and Methods}

This longitudinal prospective study was conducted at the Department of ENT, Calcutta National Medical College, from May 2017 to April 2018. In our study the patients who were undergoing total laryngectomy due to laryngeal malignancy were included. All ages and both sexes were included in the study. Our study mainly focused on the patients undergoing the esophageal speech mode of rehabilitation as opposed to those undergoing T-E puncture and TE prosthesis insertion. 


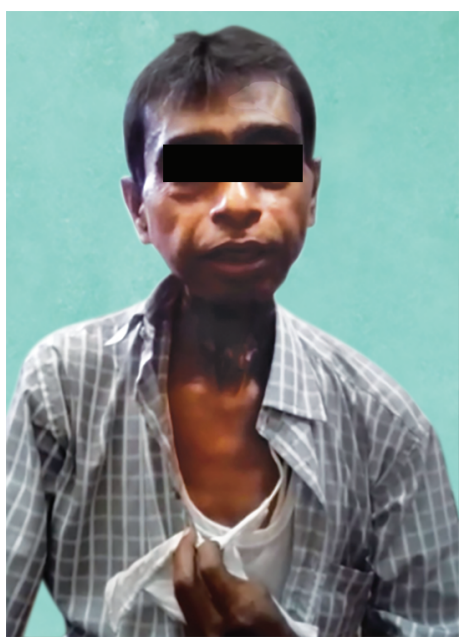

Fig. 2: Esophageal speech

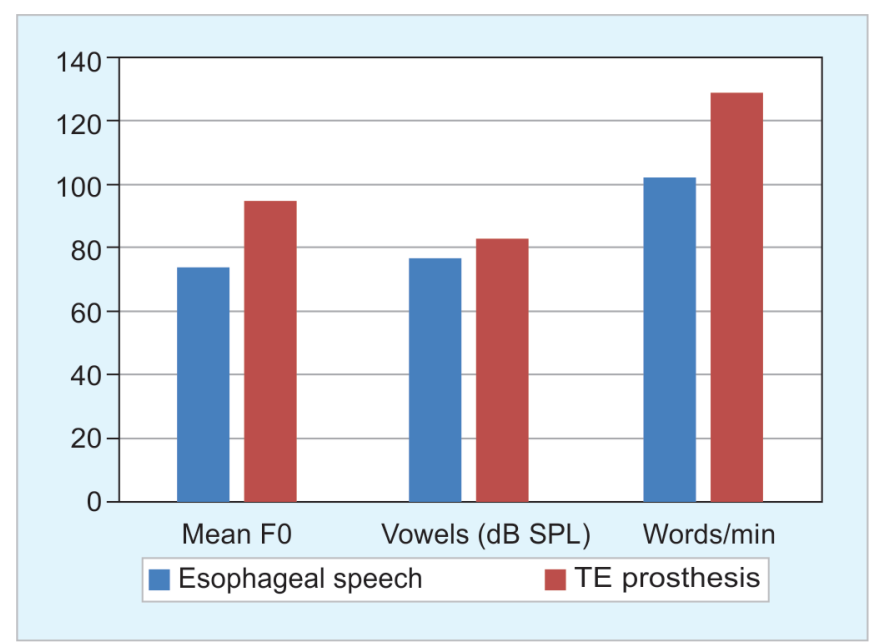

Fig. 4: Speech outcome parameters I

The outcome in both groups was assessed in terms of the following variables:

- Mean frequency for phonation

- Mean intensity for vowels

- Number of words spoken per minute

- Total number of pauses

- Number of words per phrase

- Mean maximum phonation time.

These vocal outcome parameters were accumulated and evaluated for the patients in the two groups and the final results were compared between the two groups.

\section{Results}

The analysis of speech outcome parameters in the two groups of patients revealed that the mean frequency of phonation (mean F0) in our study (Fig. 4) was $70-78 \mathrm{~Hz}$ range (average: $74 \mathrm{~Hz}$ ) in the esophageal speech group as compared to $85-104.3 \mathrm{~Hz}$ (average: $94.6 \mathrm{~Hz}$ ) in the T-E prostheses group. A similar study conducted by Robbins et al., ${ }^{10}$ had obtained values in the range pf of $65-77 \mathrm{~Hz}$ (average: $71 \mathrm{~Hz}$ ) in the esophageal speech group and $82.8-101.7 \mathrm{~Hz}$ (average: $92.2 \mathrm{~Hz}$ ) in the T-E prosthesis group. The mean intensity

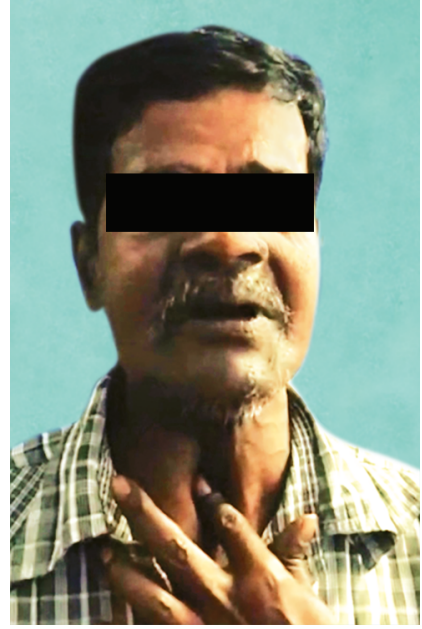

Fig. 3: Speech with TEP prosthesis

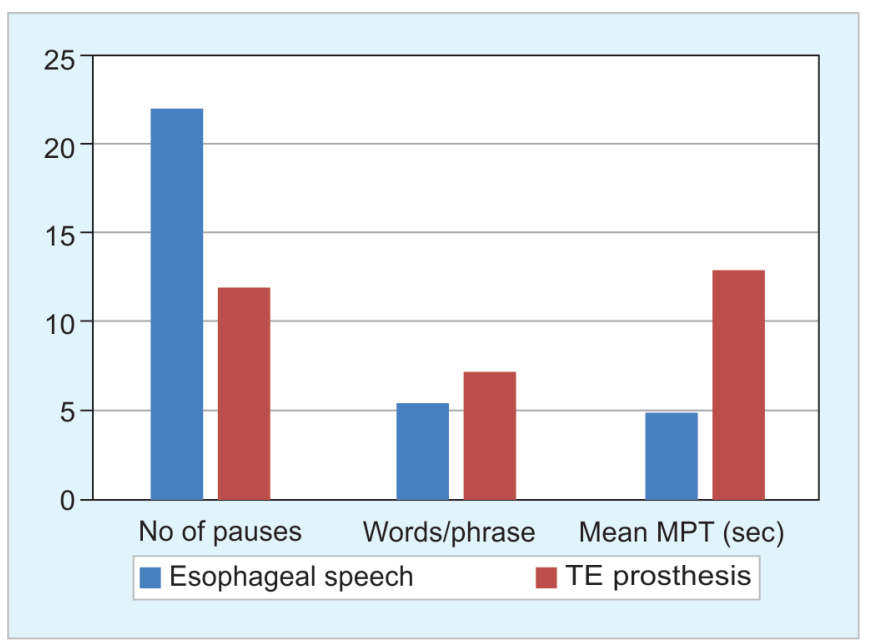

Fig. 5: Speech outcome parameters II

for vowels in our study (Fig. 4) was at $77 \mathrm{~dB}$ SPL in the esophageal speech group as opposed to $89 \mathrm{~dB}$ in the T-E prosthesis group. The study by Robbins et al. ${ }^{10}$ had obtained a value of $73.8 \mathrm{~dB}$ SPL in the esophageal speech group as opposed to $88.1 \mathrm{~dB}$ SPL in the T-E prosthesis group.

In our study the number of words per minute (Fig. 4) was 102 in the esophageal prosthesis group as against 129.5 in the T-E prosthesis group. In the study by Robbins et al. ${ }^{10}$ the values obtained were 99.1 words per minute in the esophageal speech group as against 127.5 words per minute in the T-E prosthesis group. In our study the number of pauses (Fig. 5) were 22 and 12 in the two groups, respectively. In the study by Robbins et al., ${ }^{10}$ the values obtained were 35.4 and 13.0, respectively, in the two groups.

In our study the total number of words per phrase (Fig. 5) was 5.3 and 7.2, respectively, in the esophageal speech group and T-E prosthesis group. In the study conducted by Robbins et al., ${ }^{10}$ the values in the two groups were 3 and 7.2, respectively. In our study the mean maximum phonation time (MPT) seen was 4.8 seconds and 13 seconds in the esophageal speech group and T-E prosthesis group respectively (Fig. 5). In the study done by Robbins et al., ${ }^{10}$ the values obtained were 1.9 seconds and 12.2 seconds, respectively. 


\section{Discussion}

Upon comparing the speech outcome parameters of the patients of esophageal speech with those of T-E prosthesis patients, we see that

- Mean frequency of Phonation is $78 \%$ of that seen in the T-E prosthesis group.

- Mean intensity for vowels is $86.6 \%$ of that seen in the T-E prosthesis group.

- Word production per minute is $81 \%$ of that seen in the T-E prosthesis group.

- Mean maximum phonation time is $37 \%$ of that seen in the T-E prosthesis group.

- Number of words per phrase is $73.65 \%$ of that seen in the T-E prosthesis group.

- The total no of pauses were 1.8 times that seen in the T-E prosthesis group.

- Greater patient preference was for this modality.

\section{Conclusion}

From our observations it is clear that

- With adequate training, the outcome in terms of various speech parameters in esophageal speech is significantly better the results documented from earlier studies (Video 1 ).

- The outcome parameters of esophageal speech are almost on par with that of TEP prosthesis, except the mean maximum phonation time attribute, which is still significantly higher than the corresponding previously documented values.

- In India, until an economically viable alternative becomes standardized, esophageal speech (with adequate training) is the option of choice for speech rehabilitation in post-laryngectomy patients, both due to its simplicity of use post acquisition and due to dependable results.

\section{References}

1. Lefebvre JL. Surgery for laryngopharyngeal SCC in the era of organ preservation. Clin Exp Otorhinolaryngol [Internet] 2009;2(4):159-163. DOI: 10.3342/ceo.2009.2.4.159.

2. Stankovic I, Milisavljevic M, Stankovic M. Survival after salvage total laryngectomy: the influence of previous treatment [Internet]. J Nucl Med Radiat Ther 2017;8:1-3.

3. Ashan SF, Meleca RJ, Dworkin JP. Botulinum toxin injection of the cricopharyngeus muscle for the treatment of dysphagia. Otolaryngol Head Neck Surg 2000;122(5):691-695.

4. Chao SS, Graham SM, Hoffman HT. Management of pharyngoesophageal spasm with botox. Otolaryngol Clin North Am 2004;37(3):559-566. DOI: 10.1016/j.otc.2004.01.003.

5. Karen C, Joel M. Utilization of microprocessors in voice quality improvement: the electrolarynx. Curr Opin Otolaryngol Head Neck 2000;8:138-142. DOI: 10.1097/00020840-200006000-00002.

6. Serafini I. Reconstructive laryngectomy. In: Shedd DP, Weinberg B. Surgical and prosthetic approach to speech rehabilitation. Boston: G.K. Hall; 1980. pp. 67-76.

7. Guttman MR. Rehabilitation of voice in laryngectomized patients. Arch Otolaryngol 1932;15:478-479. DOI: 10.1001/ archotol.1932.03570030496019.

8. Singer $\mathrm{ML}$, Blom PD, Hamaker RG. Voice rehabilitation after total laryngectomy. J Otolaryngol 1983;2:329-334.

9. Van Weissenbruch R, Albers FW, Bouckaert S, et al. Deterioration of the provox silicone tracheo-esophageal voice prosthesis: microbial aspects and structural changes. Acta Otolaryngol 1997;117(3): 452-458. DOI: 10.3109/00016489709113420.

10. Robbins J, Fisher HB, Blom EC, et al. A comparative acoustic study of normal, esophageal, and tracheoesophageal speech production. J Speech Hear Disord 1984;49(2):202-210. DOI: 10.1044/jshd.4902.202. 